\title{
RELAÇÃO DO SEXO E DA IDADE COM NÍVEL DE QUALIDADE DE VIDA EM RENAIS CRÔNICOS HEMODIALISADOS
}

\section{Paulo Roberto Santos}

Trabalho realizado na Unidade de Diálise e Transplante Renal da Santa Casa de Misericórdia de Sobral, Ceará, CE

\author{
Correspondência: \\ Rua Tenente Amauri Pio, \\ 380 , ap. 900 , Meireles \\ CEP 60160-090, \\ Fortaleza, CE \\ Tel: (85) 3461-1730 \\ prsantos@fortalnet.com.br
}

\begin{abstract}
RESUMO
OBjetivo. A taxa de mortalidade estacionada na última década e o aumento da prevalência de idosos entre hemodialisados motivaram o presente estudo que teve por objetivo determinar a relação do sexo e da idade com o nível de qualidade de vida (QV) em portadores de insuficiência renal crônica sob terapia hemodialítica.

Métodos. Em uma amostra de 107 indivíduos submetidos à hemodiálise regular em uma Unidade de Diálise no interior do estado do Ceará, o Medical Outcomes Study Questionaire 36-Item Short Form Health Survey (SF-36) foi utilizado para medida da QV. Foi calculado o coeficiente de correlaçãa de Pearson para estimar e testar a correlação linear entre idade e as pontuações geradas pelo SF-36, e as pontuações de acordo com o sexo foram comparadas pelo teste te de Mann-Whitney quando indicado.
\end{abstract}

REsultados. Não houve diferença entre as pontuações de acordo com o sexo. Foi encontrada correlação linear e negativa entre a idade e as dimensões de $\mathrm{QV}$ nomeadas capacidade funcional $(r=-0,289, p=0,003)$, limitação por aspectos físicos ( $r=$ $0,224, p=0,020)$, dor $(r=0,252, p=0,008)$, estado geral de saúde $(r=-0,245, p<0,00 I)$, vitalidade $(r=-0,334, p<0,00 I)$ e aspectos sociais $(r=-0,270, p=0,005)$. Não houve correlação entre idade e as dimensões limitação por aspectos emocionais $(r=-0,149, p=0,125)$ e saúde mental $(r=-0,158, p=0,103)$.

Conclusã̃o. O sexo não se relacionou com o nível de QV entre renais crônicos hemodialisados, enquanto que existiu correlação linear e negativa entre idade e as dimensões pertencentes ao aspecto físico da QV.

UnITERMOS: Insuficiência renal crônica. Hemodiálise. Qualidade de vida. Envelhecimento.

\section{INTRODUÇÃO}

Apesar das várias inovações tecnológicas incorporadas no procedimento hemodialítico, os estudos brasileiros não demonstram melhora da sobrevida dos pacientes portadores de insuficiência renal crônica (IRC) na última décadal'-3. Esse fato, que corresponde também aos registros mundiais de diálise $e^{4,5}$, impulsionou o interesse em se constatar o nível de qualidade de vida oferecida pela terapia hemodialítica, sendo essa medida cada vez mais recomendada como parâmetro a ser monitorizado regularmente. Além disso, tem crescido a importância do nível de qualidade de vida como fator prognóstico de desfechos clínicos insatisfatórios tais como: mortalidade, número de internações, falta de adesão à terapia hemodialítica ${ }^{6}$.

A falta de incremento da sobrevida dos portadores de IRC mantidos em hemodiálise poderia ser explicada pelo aumento da idade média das pessoas que têm iniciado terapia hemodialítica de manutenção nos últimos anos ${ }^{7}$. O interesse pelos efeitos da idade justifica-se pelo crescimento recente do número de idosos que iniciam terapia hemodialítica. Calcula-se que, do total aproximado de um milhão de pessoas mantidas em hemodiálise regular no mundo, mais da metade tem idade superior a 65 anos? $^{7}$.

O impacto da IRC sobre a qualidade de vida decorre de vários fatores: convívio com doença irreversível (incurável), esquema terapêutico rigoroso que provoca modificações alimentares, de hábitos, das atividades sociais e de trabalho, utilização de vários medicamentos e dependência de uma máquina ${ }^{8,9}$.

As dificuldades comuns que surgem com o avançar da idade como imobilidade, instabilidade, incontinência e dificuldade intelectual, somadas às consequêencias da terapia dialítica, tornam fundamental a pesquisa do efeito da idade sobre o nível de qualidade de vida que renais crônicos conseguem atingir sob tal tratamento. Portanto, o objetivo do estudo foi verificar de que forma o sexo e a idade de portadores de IRC submetidos à hemodiálise se correlacionam com 0 nível de qualidade de vida.

\section{Métodos}

A pesquisa foi realizada na Unidade de Diálise da Santa Casa de Sobral, Ceará, com a população de 139 indivíduos que se encontravam em tratamento hemodialítico no mês de março de 2004. Os critérios de inclusão utilizados foram: I-ser portador de IRC; 2 tempo maior do que três meses em hemodiálise; 3-não ter se submetido a transplante renal no passado; 4- idade superior a 18 anos; 5-aceitação para participar da pesquisa mediante assinatura do Termo de Consentimento Livre Esclarecido. Da população de 139 pessoas, foram excluídos 16 indivíduos com tempo em hemodiálise inferior a três meses, quatro por terem vivenciado um período como 
transplantados, seis por demonstrarem não entender os itens do instrumento utilizado na pesquisa, quatro por terem menos de 18 anos de idade. Duas pessoas se recusaram a participar do estudo. A amostra foi então constituída por 107 indivíduos.

Para medida da qualidade de vida foi utilizado como formulário o Medical Outcomes Study Questionaire 36-Item Short Form Health Survey (SF-36), instrumento do tipo genérico criado por Ware e Sherbourne ${ }^{10}$, originalmente na língua inglesa norte-americana. No Brasil, teve sua tradução e validação cultural realizada por Ciconelli" constituído de 36 itens, fornecendo pontuação em oito dimensões da qualidade de vida: capacidade funcional (CF), limitação por aspectos físicos (AF), dor (DF), estado geral de saúde (SG), vitalidade (VIT), aspectos sociais (AS), aspectos emocionais (AE) e saúde mental (SM). A pontuação varia de 0 (pior resultado) a 100 (melhor resultado).

A coleta dos dados demográficos e clínicos foi realizada tendo como fonte os registros da Unidade de Diálise. O diagnóstico da doença renal de base corresponde a critério clínico, como encontrado nos registros, e não a diagnóstico histológico.

Os dados demográficos e clínicos foram apresentados como média \pm desvio padrão e valores mínimos e máximos para as variáveis contínuas, e como percentagem para variáveis categóricas.

As pontuações geradas pelo SF-36, por meio de um sistema próprio do instrumento, foram apresentadas para cada dimensão como média \pm desvio padrão.

$\mathrm{Na}$ comparação entre as médias das pontuações geradas pelo SF-36 referentes a homens e mulheres, foi utilizado o teste t para as médias que apresentavam valores com distribuição normal, e como teste não-paramétrico o de Mann-Whitney. A verificação da distribuição dos resíduos quanto à normalidade foi realizada pelo teste de Kolmogorov-Smirnov.

Foi utilizado o coeficiente de correlação de Pearson para estimar e testar a correlação linear entre idade e as pontuações geradas pelo SF-36.

A significância estatística foi estabelecida em 5\% $(p<0,05)$.

\section{Resultados}

A amostra de 107 pessoas foi constituída por $63(58,9 \%)$ homens e 44 (4I, I\%) mulheres, com idade média de 42,9 \pm |4,7 (I8-80) anos. A cor da pele foi parda em $50(46,7 \%)$ indivíduos, branca em 44 (4I, I\%) e preta em I3 (I2, I\%). O tempo de escolaridade foi menor do que quatro anos para $72(67,3 \%)$ participantes e maior para 35 (32,7\%). A renda familiar era inferior e superior a um salário mínimo, respectivamente, para $83(77,6 \%)$ e 24 (22,4\%) pessoas. Noventa e um (85\%) indivíduos não possuíam atividade de trabalho, enquanto I6 (I5\%) trabalhavam. Quarenta e nove $(45,8 \%)$ coabitavam com três ou quatro pessoas, 39 (36,4\%) com cinco ou mais, e $19(17,8 \%)$ com uma ou duas pessoas.

As principais causas da IRC foram: glomerulonefrite em 50 indivíduos (46,7\%) e hipertensão em 34 (31,8\%). As demais causas foram distribuídas entre diabetes ( $N=5,4,7 \%$ ), doença policística do adulto ( $N=5,4,7 \%)$, lúpus eritematoso sistêmico $(N=5,4,7 \%)$, pielonefrite crônica ( $N=4,3,7 \%)$ e nefropatia obstrutiva $(N=4,3,7 \%)$. O tempo médio de manutenção em hemodiálise da amostra de foi de 47,4 \pm 43,8 (3-191) meses.
Em ordem decrescente, as médias das pontuações referentes à qualidade de vida nas diversas dimensões englobadas pelo instrumento SF-36 foram: $62 \pm 22,6$ para SM; $61,7 \pm 27,8$ para AS; 54,9 $\pm 28,2$ para DF; $53,4 \pm 27, I$ para CF; $49,8 \pm 23$ no que se refere à VIT; 40,5 \pm 23 para SG; 34,7 \pm 4 I, 4 para AE e $33 \pm 4$ I, I para AF.

Segundo o sexo, as médias das pontuações referentes aos homens $(\mathrm{N}=63)$ e mulheres $(\mathrm{N}=44)$ foram, respectivamente: $53,5 \pm 27,5$ versus $52,9 \pm 26,6$ para $C F(p=0,902) ; 33,4 \pm 42,9$ versus $31,6 \pm$ 38,9 para AF $(p=0,824) ; 56,6 \pm 26$, Iversus $52,3 \pm 30,8$ para a dimensão DF $(p=0,429) ; 41, I \pm 23,2$ versus $40,2 \pm 23$ para SG $(p=0,827) ; 49,3 \pm 24,3$ versus $50,5 \pm 21,1$ para VIT $(p=0,792) ; 63,1$ $\pm 27,5$ versus $59,2 \pm 28,3$ para AS ( $p=0,475) ; 34,8 \pm 40,6$ versus $35,4 \pm 42,8$ para AE ( $p=0,938)$; e $62,9 \pm 22,9$ versus $60,9 \pm 22,1$ para SM $(p=0,650)$.

Os coeficientes de correlação de Pearson entre idade e as dimensões da qualidade de vida foram: $-0,289(p=0,003)$ para $C F,-0,224$ $(p=0,020)$ para $A F,-0,252(p=0,008)$ em relação à dimensão $D F$, $-0,245(p=0,0 I I)$ referente ao SG, $-0,334(p<0,00 I)$ para VIT, $0,270(p=0,005)$ para $A S,-0,149(p=0,125)$ concernente à AE e $0,158(p=0,103)$ para SM.

\section{Discussão}

A dimensão de qualidade de vida com pontuação mais baixa (pior resultado) foi a de limitação por aspectos físicos. Esse resultado é concordante com a literatura nacional, que demonstra predomínio de pontuações mais baixas obtidas pelo SF-36 referentes a dimensão limitação por aspectos físicos entre pacientes renais crônicos submetidos a esquema regular de hemodiálise em diversas unidades de diálise ${ }^{12-15}$. A idade média da amostra em nosso estudo, compreendida em faixa potencialmente produtiva, torna esse achado estratégico: por poder explicar a baixa ocupação profissional e por tornar-se alvo de máxima atenção por parte dos profissionais de saúde envolvidos no cuidado desses pacientes. Existem evidências na literatura mundial de melhora do aspecto físico da qualidade de vida em hemodialisados após implantação de programa de prática regular de exercícios ${ }^{16-18}$

Em análise univariada previamente realizada, nenhuma das variáveis sociodemográficas (cor da pele, escolaridade, renda familiar, atividade profissional, número de coabitantes) demonstrou diferença estatisticamente significante em relação às pontuações geradas pelo SF36. Provavelmente, isto se deve ao número pequeno associado à homogeneidade do perfil social da amostra, constituída em sua totalidade por clientela do Sistema Único de Saúde e residente no interior do estado do Ceará, que impossibilitou poder estatístico para demonstrar possíveis diferenças da qualidade de vida dependentes das variáveis sociais. Por esta razão, a relação entre sexo e idade e nível de qualidade de vida não foi submetida à análise multivariada com ajuste pelas demais variáveis.

Não foi constatada diferença estatisticamente significante entre as pontuações no que se refere ao sexo na nossa amostra. Em países como Canadá, Estados Unidos e Inglaterra, onde o 
SF-36 foi utilizado para gerar um normograma populacional (de pessoas saudáveis) do nível de qualidade de vida, foi evidenciado que homens obtêm maior pontuação do que mulheres em todas oito dimensões do teste, a não ser na Inglaterra onde a diferença ocorreu em sete das oito dimensões ${ }^{|9-2|}$.

Entre hemodialisados, em concordância com os nossos resultados, Kalantar-Zadeh et al. ${ }^{22}$ também não encontraram diferença no que se refere ao sexo em quaisquer das pontuações geradas pelo SF-36.

O SF-36 permite gerar de modo resumido pontuações referentes a apenas dois aspectos: físico e mental. A pontuação desses aspectos não deriva simplesmente da média das pontuações referentes às dimensões incluídas em cada aspecto; há ponderação diferente para a contribuição de cada dimensão para a pontuação final dos dois aspectos. $O$ aspecto físico sendo determinado pelas dimensões: capacidade funcional e limitação por aspectos físicos, principalmente; e menos pela dor e estado geral de saúde. O aspecto mental inclui: saúde mental, que tem ponderação maior e predomina como influenciador da pontuação deste aspecto, seguido em ordem decrescente de importância por aspectos emocionais, aspectos sociais, vitalidade. A dimensão vitalidade também contribui para pontuação do aspecto físico, assim como estado geral de saúde contribui para a pontuação resumida como aspecto mental. Alguns trabalhos na literatura têm seus resultados sobre qualidade de vida analisados por meio destas pontuações resumidas, referentes aos dois aspectos.

Em nosso estudo, foi observada correlação linear negativa da idade com as dimensões dor, estado geral de saúde, vitalidade, aspectos sociais, capacidade funcional e aspectos físicos. A correlação foi fortemente negativa para as dimensões dor $(p=0,008)$, vitalidade $(p<0,00 \mathrm{I})$, aspectos sociais $(p=0,005)$ e capacidade funcional $(0,003)$. A idade não apresentou correlação com saúde mental e aspectos emocionais. Sendo assim, o predomínio de correlação negativa da idade é com as dimensões compreendidas como do aspecto físico da qualidade de vida. Portanto, o resultado leva a reflexão de que o avançar da idade compromete mais o aspecto físico e menos o mental. Nosso achado é extensamente corroborado tanto por estudos com pessoas saudáveis como por aqueles com portadores de IRC, como descrito a seguir.

As enquetes populacionais que utilizaram o SF-36 para gerar normogramas das pontuações referentes à qualidade de vida demostraram em países como Estados Unidos ${ }^{19}$, Suécia ${ }^{23}$, Canadáa ${ }^{21}$ e Holanda ${ }^{24}$ o mesmo padrão de declínio gradual das pontuações, ao longo dos anos, das dimensões referentes predominantemente ao aspecto físico, em comparação ao mental.

Especificamente, a falta de correlação da idade com saúde mental encontrada em nosso estudo é constatada por Singer, Hopman e MacKenzie ${ }^{25}$. Esses autores identificaram igualmente na população geral e em doentes crônicos (incluídos transplantados renais, hemodialisados e portadores de esclerose múltipla) declínio da capacidade funcional (pelo SF-36) com o avançar da idade e manutenção da dimensão saúde mental.

Mais impressionante é o resultado em relação a saúde mental de idosos hemodialisados de Lamping et al..$^{26}$, que focaram a pesquisa em idosos com mais de 70 anos mantidos sob terapia dialítica (hemodiálise e diálise peritonial). Os autores utilizaram o SF-36 como instrumento de medida da qualidade de vida, e compararam os resultados do grupo de renais crônicos dialisados com a população geral, utilizando parâmetros populacionais norte-americano ${ }^{27} \mathrm{e}$ inglês ${ }^{28}$. O resultado foi igual qualidade de vida no que se refere à dimensão saúde mental dos dialisados quando comparado à população geral, exceto pelo rebaixamento, em relação aos primeiros, da qualidade de vida no seu aspecto físico.

Como seria esperado, há resultados conflitantes quanto à correlação da idade com o nível de qualidade de vida medido pelo SF-36. $\mathrm{Na}$ comparação de dois grupos de sujeitos (idade superior versus inferior a 65 anos) mantidos em diálise e também transplantados renais, Rebollo et al..$^{29}$ encontraram maior pontuação nas dimensões capacidade funcional, limitação por aspectos físicos e estado geral de saúde no grupo dos idosos quando comparados aos hemodialisados mais jovens. Ao compararem transplantados com mais e menos de 65 anos, confirmaram a superioridade da qualidade de vida no grupo dos idosos nas dimensões correspondentes a capacidade funcional, dor, estado geral de saúde, aspectos sociais, limitação por aspectos emocionais e saúde mental.

No Brasil, Chang et al..$^{30}$ não encontraram diferença de qualidade de vida em quaisquer dos aspectos englobados pelo SF-36 ao comparar dois grupos de pessoas submetidas à hemodiálise: com idade superior e inferior a 60 anos.

Sendo assim, não há consenso nos resultados das pesquisas sobre a influência da idade na qualidade de vida. De qualquer modo, o mais importante é que cada unidade de diálise monitorize o nível de qualidade de vida dos pacientes com a intenção de realizar intervenções que revertam as dimensões comprometidas. Diante da nossa constatação de que a dimensão limitação por aspectos físicos é a mais afetada de maneira geral na amostra, e de que a idade compromete mais marcantemente as dimensões do componente físico, os renais crônicos com mais idade devem ter especial atenção quanto a reduzir fatores que restringem as atividades físicas realizadas no dia-a-dia, seja com fisioterapia, programação de exercícios físicos ou intervenção específica em patologias articulares e/ou sistêmicas.

\section{Conclusão}

Não houve diferença no nível de qualidade de vida no que se refere ao sexo. Porém, houve correlação linear e negativa entre idade e as seguintes dimensões de qualidade de vida: capacidade funcional, limitação por aspectos físicos, dor, estado geral de saúde, vitalidade e aspectos sociais. As dimensões sem correlação com a idade foram: limitação por aspectos emocionais e saúde mental. Conclui-se que o aspecto físico da qualidade de vida nesse grupo de pacientes é mais afetado com o avançar da idade do que o aspecto mental.

\section{Conflito de interesse: não há}

\section{SUMMARY}

Relationship between gender and age With Quality of LIFE IN CHRONIC HEMODIALYSIS PATIENTS

BACKGROUND. The stagnant mortality rate of the last decade and the increased prevalence of aged people among hemodialysis patients 
motivated the present study to determine the relation of gender and age with the quality of life (QL) of patients with chronic kidney failure treated with hemodialysis.

METHODS. From the sample of 107 individuals who underwent regular hemodialysis in a Dialysis Unit in the interior of the state of Ceará, Brazil, the Medical Outcomes Study Questionnaire 36-Item Short Form Health Survey (SF-36) was used to assess quality of life. Pearson's correlation coefficient was used to calculate and to test the linear correlation between age and the score generated by the SF-36. The score for gender was compared by the t test and the Mann-Whitney when indicated.

RESULTS. There was no difference between the score related to gender. There was no negative and linear correlation between age and the dimensions of QL named: Physical Functioning ( $r=-0.289, p=0.003)$, Physical Role $(r=-0.224, p=0.020)$, Body Pain $(r=0.252, p=0.008)$, General Health $(r=-0.245, p<0.001)$, Vitality $(r=-0.334, p<0.001)$ and Social Functioning $(r=-0.270, p=0.005)$. There was no correlation between age and the dimensions: Role of Emotions $(r=-0.149, p=0.125)$ and Mental Health $(r=-0.158, p=0.103)$.

CONCLUSION. Gender did not correlate with the level of QL among chronic hemodialysis patients, while there was a linear and negative correlation between age and the dimensions relating to the physical aspect of the QL. [Rev Assoc Med Bras 2006, 52(5): 356-9]

KEY wORDS: Chronic kidney failure. Hemodialysis. Quality of life. Aging.

\section{REFERÊNCIAS}

I. Andrade LGM, Gabriel DP, Martin LC, Cruz AP, Balbi AL, Barretti P. Sobrevida em hemodiálise no Hospital de Botucatu - UNESP: comparação entre a primeira e a segunda metades da década de 90. J Bras Nefrol 2005;27( I): I-7.

2. Martin LC, Caramori JC, Felippe MJDB, Baretti P. Sobrevida em hemodiálise nos últimos dez anos no Hospital das Clínicas da Faculdade de Medicina de Botucatu: comparação entre a primeira e segunda metades da década. J Bras Nefrol 2000;22(supl 3):93.

3. Lima JJG, Fonseca JA, Godoy ADDialysis, time and death: comparisons of two consecutive decades among patients treated at the same Brazilian dialysis center. Braz J Med Biol Res 1999;32(3):289-95.

4. Report on management of renal failure in Europe, XXIV, 1993. Nephrol Dial Transplant 1993; 10(5).

5. Marcelli D, Satannard D, Conte F, Held PJ, Locatelli F. ESRD patient mortality with adjustment for comorbid conditions in Lombardy (Italy) versus United States. Kidney Int 1996;50: I 0 I 3-8.

6. Merkus MP, Jager KJ, Dekker FW, Haan RJ, Boeschoten EW, Krediet RT. Predictors of poor outcome in dialysis patients: The Netherlands Cooperative Study on the Adequacy of Dialysis. Am J Kidney Dis 2000;35(I):66-79.

7. Sims RJA. The increasing number of older patients with renal disease. BMJ 2003;327:463-64.

8. Lima AFC, Gualda DMR. Reflexão sobre a qualidade de vida do cliente renal crônico submetido a hemodiálise. Nursing 2000;20-3.

9. Silva DMG, Vieira RM, Koschnik Z, Azevedo M, Souza SS. Qualidade de vida de pessoas com insuficiência renal crônica em tratamento hemodialítico. Rev Bras Enfermagem 2002;55(5):562-67.

10. Ware JE, Sherbourne CD. The MOS 36-Item Short-Form Health Survey (SF-36). I. Conceptual framework and item selection. Med Care 1992:30:473-83.

I I. Ciconelli RM. Tradução para o português e validação do questionário de avaliação de qualidade de vida "Medical outcomes study 36-item short form health survey (SF-36)" [tese]. São Paulo:Universidade Federal de São Paulo; 1997.
12. Neto JFR, Ferraz M, Cendoroglo S, Draibe L, Yu L, Sesso R. Quality of life at the initiation of maintenance dialysis treatment: a comparison between the SF-36 and the KDQ questionnaires. Qual Life Res 2000;9: I 0 I-7.

13. Neto JF, Sesso R. Nível sócio-econômico e a qualidade de vida de pacientes com insuficiência renal crônica terminal no início do tratamento por hemodiálise. J Bras Nefrol 2000;3(supl 3):94.

14. Castro M, Caiuby AVS, Draibe SA, Canziani MEF. Qualidade de vida de pacientes com insuficiência renal crônica em hemodiálise avaliada através do instrumento genérico SF-36. Rev Assoc Med Bras 2003;49:245-9.

15. Pereira LC, Chang J, Fadil-Romão MA, Abensur H, Araújo MRT, Noronha IL, CampagnariJC, RomãoJrJE. Qualidade de vida relacionada à saúde em paciente transplantado renal. J Bras Nefrol 2003;25: 10-6.

16. Merkus MP, Jager KJ, Dekker FW, Boeschoten EW, Stevens P, Krediet RT. Quality of life in patients on chronic dialysis: self-assessment 3 months after the start of treatment. Am J Kidney Dis 1997;29:584-92.

17. Oh-Park M. Exercise for the dialyzed: aerobic and strength training during hemodialysis. Am J Phys Med Rehabil 2002;81:814-21.

18. Pugh-Clarke K, Koufaki P, Rowley V, Mercer T, Naish P. Improvement in quality of life of dialysis patients during six months of exercise. EDTNA ERCA J 2002;28: I I-2.

19. Ware JE, Snow KK, Kosinski M, Gandek B. SF-36 manual and interpretation guide. Boston: The Health Institute, New England Medical Center; 1993

20. Jenkinson C, Stewart-Brown S, Petersen S, Paice C. Assessment of the SF36 version 2 in the United Kingdom. J Epidemiol Commmunity Health 1999:53:46-50.

21. Hopman WM, Towheed T, Anastassiades T, Tenehouse A, Poliquin S, Berger C, et al. Canadian normative data for the SF-36 health survey. Can Med Assoc J 2000; 1 63:265

22. Kalantar-Zadeh K, Kopple JD, Block G, Humphreys MH. Association among SF36 quality of life measures and nutrition, hospitalization, and mortality in hemodialysis. J Am Soc Nephrol 2000 I; I 2:2797-806.

23. Sullivan M, Karlsson J. The Swedish SF-36 Health survey III. Evaluation of criterion-based validity: results from normative population. J Clin Epidemiol I 998;5 I: I I05- I3.

24. Aaronson NK, Muller M, Cohen PD, Essink-Bot ML, Fekkes M, Sanderman R. Translation, validation and norming of the Durch language version of the SF-36 Health survey in community and chronic disease population. J Clin Epidemiol 1998;51: 1055-68.

25. Singer MA, Hopman WM, MacKenzie TA. Physical functioning and mental health in patients with chronic medical conditions. Qual Life Res | 999:8:687-91.

26. Lamping DL, Constantinovici N, Roderick P, Normand C, Henderson L, Harris S, et al. Clinical outcomes, quality of life, and costs in the North Thames dialysis Study of elderly people on dialysis: a prospective cohort study. Lancet 2000;356: I 543-50.

27. Ware JE, Kosinski MA, Keller SD. SF-36 physical and mental component summary measures: a user's manual. Boston: The Health Institute, New England Medical Center; 1994

28. Bowling A, Bond M, Jenkinson C, Lamping DL. Short Form 36 (SF-36) Health Survey questionaire: which normative data should be used? Comparisons between the norms provided by the Omnibus Survey in Britain, the Health Survey for England and the Oxford Health and Lifestyle Survey. J Public Health Med 1999;21:255-70.

29. Rebollo P, Ortega F, Baltar JM, Álvarez-UF, Navascués R, Álvarez-Grande $\mathrm{J}$. Is the loss of health-related quality of life during renal replacement therapy lower in elderly patients than in younger patients? Nephrol Dial Transplant 2000 I ; 6: 1 675-80.

30. Chang J, Pereira LC, Abensur H, Araújo MRT, Noronha IL, Romão Jr JE. Análise da qualidade de vida no paciente em hemodiálise. J Bras Nefrol 2002;24(supl 2): 160.

Artigo recebido: 13/06/2005

Aceito para publicação: 03/I I/2005 